\title{
Phosphonate Metal-Organic Frameworks: A Novel Family of Semiconductors
}

\author{
Konrad Siemensmeyer, Craig A. Peeples, Patrik Tholen, Franz-Josef Schmitt, \\ Bünyemin Çoşut, Gabriel Hanna,** and Gündoğ Yücesan**
}

Herein, the first semiconducting and magnetic phosphonate metal-organic framework (MOF), TUB75, is reported, which contains a 1D inorganic building unit composed of a zigzag chain of corner-sharing copper dimers. The solid-state UV-vis spectrum of TUB75 reveals the existence of a narrow bandgap of $1.4 \mathrm{eV}$, which agrees well with the density functional theory (DFT)calculated bandgap of $1.77 \mathrm{eV}$. Single-crystal conductivity measurements for different orientations of the individual crystals yield a range of conductances from $10^{-3}$ to $10^{3} \mathrm{~S} \mathrm{~m}^{-1}$ at room temperature, pointing to the directional nature of the electrical conductivity in TUB75. Magnetization measurements show that TUB75 is composed of antiferromagnetically coupled copper dimer chains. Due to their rich structural chemistry and exceptionally high thermal/ chemical stabilities, phosphonate MOFs like TUB75 may open new vistas in engineerable electrodes for supercapacitors.

geometries and functional groups, and the option of postsynthetic modification of pore surfaces, ${ }^{[8-10]}$ many diverse applications of MOF chemistry have emerged, including small molecule storage, ${ }^{[11]}$ greenhouse gas sequestration, ${ }^{[12]}$ drug delivery, ${ }^{[13,14]}$ and detoxifying agents, ${ }^{[15]}$ and the presence of IBUs has led to applications in heterogeneous catalysis, ${ }^{[16,17]}$ magnetism, ${ }^{[18-21]}$ and conductivity. ${ }^{[21,22]}$ Among these applications, magnetic MOFs have recently attracted a great deal of attention due to the possibility of creating tunable magnetic materials by varying the host-guest interactions at pore sites or exploiting structural changes induced by MOF breathing; and conductive MOFs are expected to serve as next-generation

Metal-organic frameworks (MOFs) emerged as a new family of microporous materials at the beginning of the 21st century. ${ }^{[1-5]}$ They are composed of inorganic building units (IBUs) and organic linkers, which combine to create microporous frameworks. ${ }^{[1,3,6,7]}$ Owing to the vast range of organic linker

Dr. K. Siemensmeyer

Helmholtz-Zentrum

Berlin 14109, Germany

C. A. Peeples, Prof. G. Hanna

University of Alberta

Edmonton T6C 2R3, Canada

E-mail: gabriel.hanna@ualberta.ca

P. Tholen, Prof. G. Yücesan

Technische Universität

Berlin 13355, Germany

E-mail: yuecesan@tu-berlin.de

F.-J. Schmitt

Marthin-Luther-Universität Halle-Wittenberg

Halle (Saale) 06120, Germany

B. Çoşut

Gebze Technical University

Gebze 41400, Turkey

The ORCID identification number(s) for the author(s) of this article can be found under https://doi.org/10.1002/adma.202000474.

(C) 2020 The Authors. Published by WILEY-VCH Verlag GmbH \& Co. $\mathrm{KGaA}$, Weinheim. This is an open access article under the terms of the Creative Commons Attribution-NonCommercial-NoDerivs License, which permits use and distribution in any medium, provided the original work is properly cited, the use is non-commercial and no modifications or adaptations are made.

DOI: 10.1002/adma.202000474 porous electrode materials with higher and customizable surface areas compared to active carbon electrodes. ${ }^{[21-23]}$

Traditional MOFs have primarily relied on molecular IBUs known widely as paddle wheel patterns. ${ }^{[1-3]}$ To synthesize magnetic MOFs based on molecular IBUs, the IBUs must be close enough to each other to generate the desired magnetic interactions. The design patterns for such magnetic MOFs have been summarized in two recent review articles. ${ }^{[18,19]}$ Typically, shorter linkers such as $\mathrm{CN}$ or azolate linkers make magnetic interactions possible between the inorganic components. ${ }^{[24,25]}$ Also, linkers that can generate free radicals may be used to create magnetically significant MOFs. ${ }^{[26,27]}$ However, because MOF chemistry has evolved toward the use of longer organic linkers for larger surface areas, the distances between the molecular IBUs has increased and thereby diminished the possibility of constructing magnetic MOFs with molecular IBUs. ${ }^{[28]}$ Thus, new architectural strategies for synthesizing magnetic MOFs are in need. Along these lines, 1D and 2D IBUs can provide a more suitable platform for magnetic interactions as the metal centers may come into close proximity to each other in such geometries. ${ }^{[29-31]} \mathrm{MOFs}$, which are synthesized at high temperatures and under hydrothermal reaction conditions, usually form 1D chain IBUs. ${ }^{[32-34]}$ In contrast, conventional MOFs, which are synthesized at low temperatures in the presence of organic solvents, ${ }^{[1-6]}$ usually form molecular IBUs. 1D magnetic chains are well known, ${ }^{[29]}$ but relatively few porous magnetic MOFs with 1D IBUs have been reported in the literature. ${ }^{[18,19,25,31,35]}$

The construction of conductive MOFs requires more subtle design elements. For example, highly conjugated linkers such as phthalocyanine or porphyrins with orthodiimine, 


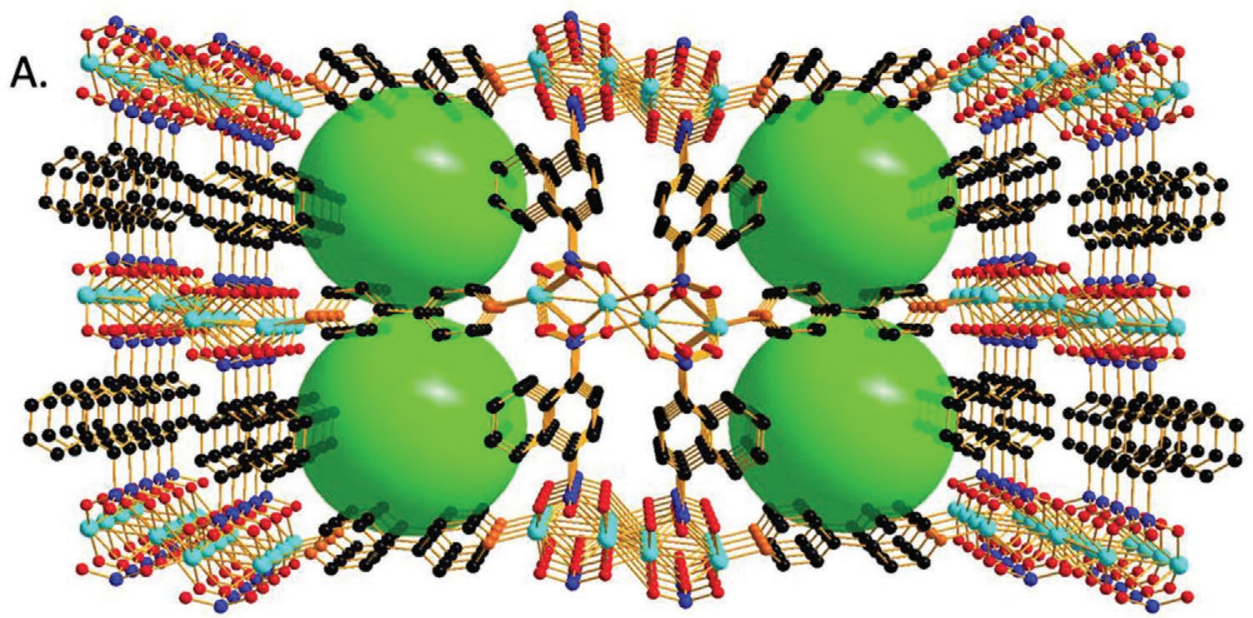

B.

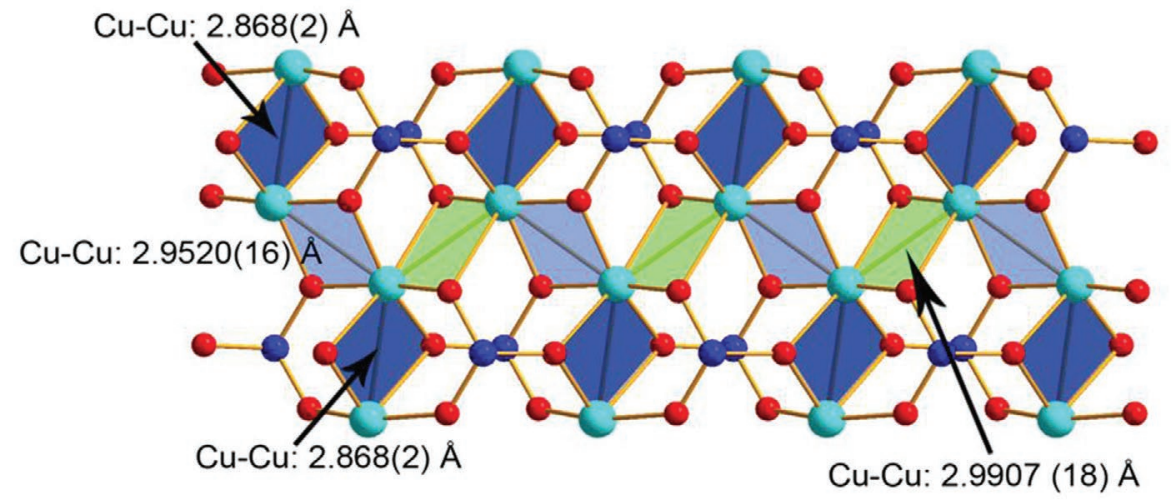

Figure 1. A) One layer of the $\left[\left\{\mathrm{Cu}_{2}\left(4,4^{\prime} \text {-bpy }\right)_{0.5}\right\}\right.$ (1,4-NDPA) (TUB-75) MOF, showing nine 1D copper dimer IBUs and four void channels (which extend into and out of plane). B) Side view of the ID IBU consisting of a zigzag chain of corner-sharing copper dimers, with Cu-Cu distances of less than $3 \AA$. Dimers are colored based on their $\mathrm{Cu}-\mathrm{Cu}$ bond distances. Color definitions: $\mathrm{O}$-red; $\mathrm{N}$-orange; $\mathrm{Cu} \longrightarrow$ cyan; $\mathrm{C}$-black; P—blue.

orthodihydroxy, and azolate metal-binding units connected via molecular IBUs composed of a single metal ion, are known to give rise to conductivity. ${ }^{[21-23]}$ However, due to the limited number of metal-binding modes for the single nitrogen and oxygen donating linkers and the highly conservative nature of metal binding in these systems, progress in the design of conductive MOFs has been limited. Alternative metal binding units capable of yielding both rich structural diversity and conductivity are needed for constructing next-generation conductive MOFs. ${ }^{[34,36]}$

Phosphonate MOFs are known for their high structural diversity due to the multiple metal-binding modes and protonation states of the phosphonic acid group. ${ }^{[37]}$ They are known to contain complex molecular clusters and 1D/2D IBUs. ${ }^{[30,33-36]}$ Recently, Yücesan and co-workers synthesized the phosphonate MOF TUB75 (where TUB stands for Technische Universität Berlin) at temperatures above $180{ }^{\circ} \mathrm{C}$ and under hydrothermal reaction conditions. ${ }^{[38]}$ The crystal structure revealed that this MOF contains 1D copper dimer chains linked by polyaromatic 1,4-naphthalenediphosphonic acid linkers (see Figure 1). This chain structure is unique compared to that of previously reported 1D IBUs in the literature with respect to the presence of three different (and relatively short) characteristic $\mathrm{Cu}-\mathrm{Cu}$ distances along the 1D IBU (see Figure 1B). ${ }^{[30,33-36]}$ As seen in Figure 1B, TUB75 is composed of zigzag copper dimer chains (one of which is portrayed in Figure 1B; the experimental and calculated $\mathrm{Cu}-\mathrm{Cu}$ distances are given in Figure S2 and Table S1, Supporting Information, respectively). Its surface area was previously measured to be $132.1 \mathrm{~m}^{2} \mathrm{~g}^{-1}$. ${ }^{[38]}$ The thermal decomposition patterns of phosphonate MOFs constructed using 4,4'-bipyridine as the auxiliary linker with 1,4-naphthalenediphosphonic acid, 2,6-naphthalendiphosphonic acid, and different aromatic phosphonic acids were also previously reported. ${ }^{[38,39]}$ MOFs in this family have a general tendency to be thermally stable up to $\approx 375{ }^{\circ} \mathrm{C}$, after which thermal decomposition begins. In our previous work with 2,6-naphthalendiphosphonic acid, we found that removing the $4,4^{\prime}$-bipyridines to obtain pure metal phosphonates increases the thermal stability to $\approx 400^{\circ} \mathrm{C} .{ }^{[37]}$ As seen in Figure $1 \mathrm{~B}$, all of the 1,4naphthalenediphosphonic acids in TUB75 are fully deprotonated, which leads to substantial electron delocalization within the 1D IBU (see electronic population analyses in Table S2 of the Supporting Information).

In light of the above, in this study, we revisit our phosphonate MOF TUB75 to explore its conductive and magnetic properties. As we will discuss below, we find that TUB75 has an indirect bandgap of $1.4 \mathrm{eV}$ (based on a Tauc plot of the UVVis spectrum), making it a semiconductor. In addition, we find that TUB75 possesses an antiferromagnetic chain-type IBU. Our density functional theory (DFT) calculations of TUB75's bandgap, band structure, partial density of states, and relative energies of the ferromagnetic (FM) and antiferromagnetic 


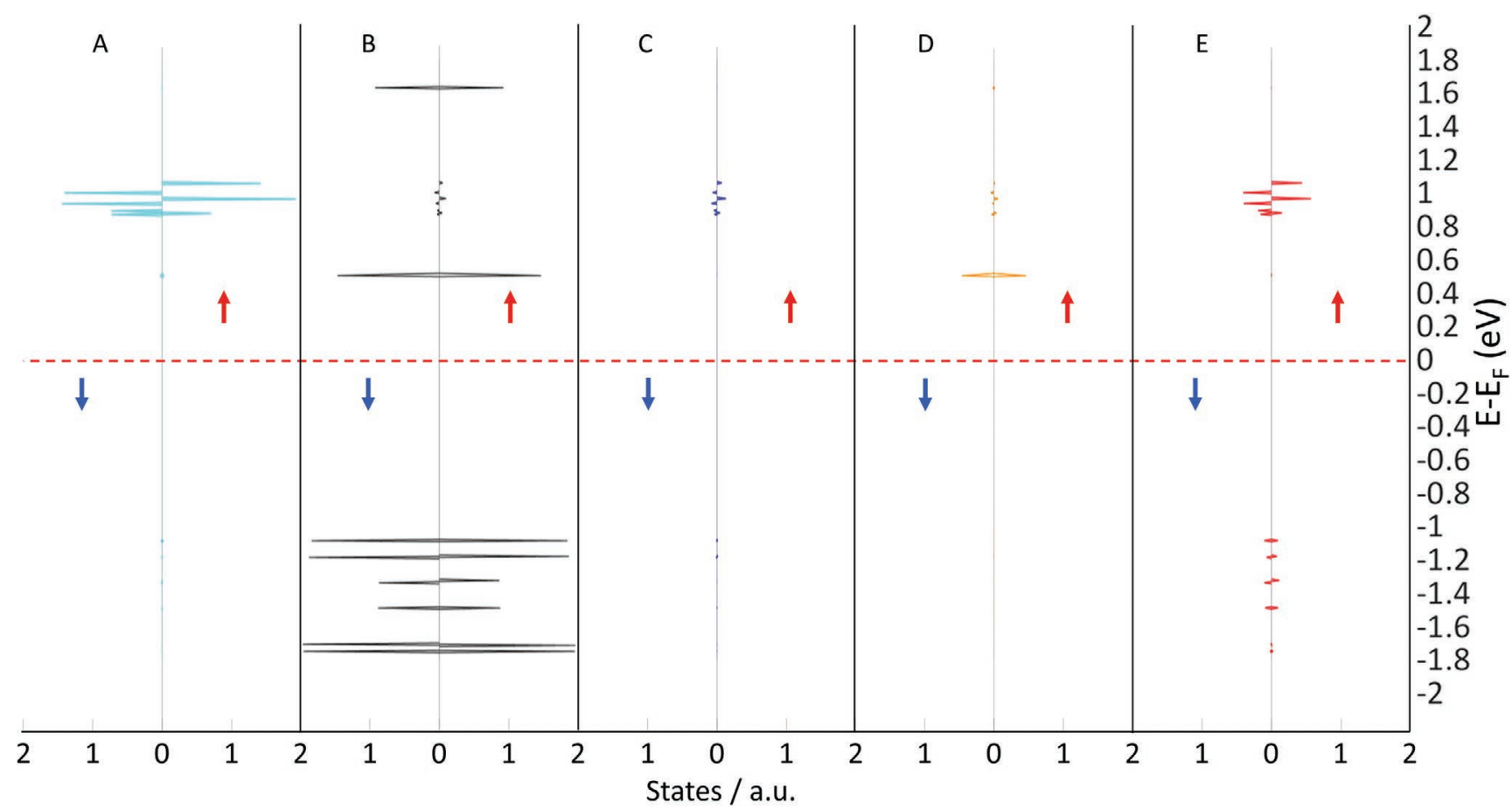

Figure 2. Spin-up and spin-down projected density of states for TUB75 in the AFM configuration. A) Copper. B) Carbon. C) Nitrogen. D) Phosphorous. E) Oxygen.

(AFM) configurations, provide detailed insight into its electronic structure. The details of our calculations, which employed Slater-type orbital (STO) basis sets, can be found in the Computational Details section of the Supporting Information.

In Figure 3B, we present an indirect Tauc plot derived from the UV-vis spectrum (shown in Figure S2, Supporting Information) of a sample of pure handpicked (under a microscope) TUB75 crystals-thin green needles with an average length of $0.5 \mathrm{~mm}$. Linear extrapolation of this Tauc plot ${ }^{[40,41]}$ yields an estimate of the optical bandgap of $1.4 \mathrm{eV}$, indicative of a semiconductor. To shed light on the origin of the semiconductivity, we performed DFT calculations, the details of which are provided in the Supporting Information. The density of states calculation yielded a HOMO-LUMO gap of $E_{\mathrm{g}}=1.77 \mathrm{eV}$, which is in good agreement with the experimental bandgap of $1.4 \mathrm{eV}$. Based on the projected density of states (pDOS) in Figure 2, we see that the HOMO-LUMO gap is predominantly due to atomic orbitals associated with the carbon atoms in the $\pi$-conjugated 1,4-naphthalenediphosphonic acid (1,4-NDPA) and 4,4'-bipyridine (4,4'-bpy) auxiliary linker groups. It also appears that there is some contribution from the nitrogen orbitals to the LUMO and a very small contribution from the oxygen orbitals to the HOMO. As for copper, there is effectively no contribution from the copper orbitals to the HOMO and LUMO. There is indication of spin dependence in the higher energy virtual orbitals, primarily associated with the copper atoms and smaller contributions coming from carbon, nitrogen, phosphorous, and oxygen. We further projected the carbon pDOS into the individual contributions from the 1,4-NDPA and the 4,4'-bpy carbons (Figure 3). This projection reveals that the HOMO and LUMO are spatially separated, with the HOMO localized on the 1,4-NDPA carbons and the
LUMO localized on the 4,4'-bpy carbons. In such a case, a photoexcited electron (in the LUMO) would be spatially separated from its hole (in the HOMO).

Electrical conductivity measurements on MOFs have been mainly based on polycrystalline pellets. However, such measurements may greatly underestimate the conductance of the MOF due to contact/grain boundary resistances and anisotropic electrical conduction. On the other hand, single-crystal measurements can provide much more accurate conductance values, provided that the crystals are large enough. In light of this, we carried out a number of single-crystal measurements on TUB75 by clamping the individual crystals between two gold surfaces of a relay. From room-temperature measurements, we obtained a range of resistances from $10 \Omega$ to $10 \mathrm{M} \Omega$, depending on the orientation of the crystal with respect to the gold surfaces. Assuming that the TUB75 crystal makes perfect contact with the gold surfaces, these resistances yield a maximum conductance of $\approx 10^{3} \mathrm{~S} \mathrm{~m}^{-1}$ and a minimum conductance of $\approx 10^{-3} \mathrm{~S} \mathrm{~m}^{-1}$ (see Supporting Information for the details of the calculations). However, since the TUB75 crystals do not make perfect contacts with the gold surfaces, the actual conductances could even be higher than our reported values. Nevertheless, our results show that TUB75 is a semiconductor and provide strong evidence of the directional nature of the electrical conductivity of TUB75. We are currently working on growing larger crystals to maximize the contact surface area in order to better understand TUB75's directional conductivity

Next, we report the results of our magnetization measurements on TUB75 in Figure 4A (see Supporting Information for the details of the measurements). The magnetization data exhibits a Néel temperature of $T \cong 30 \mathrm{~K}$ (i.e., the temperature corresponding to the maximum 


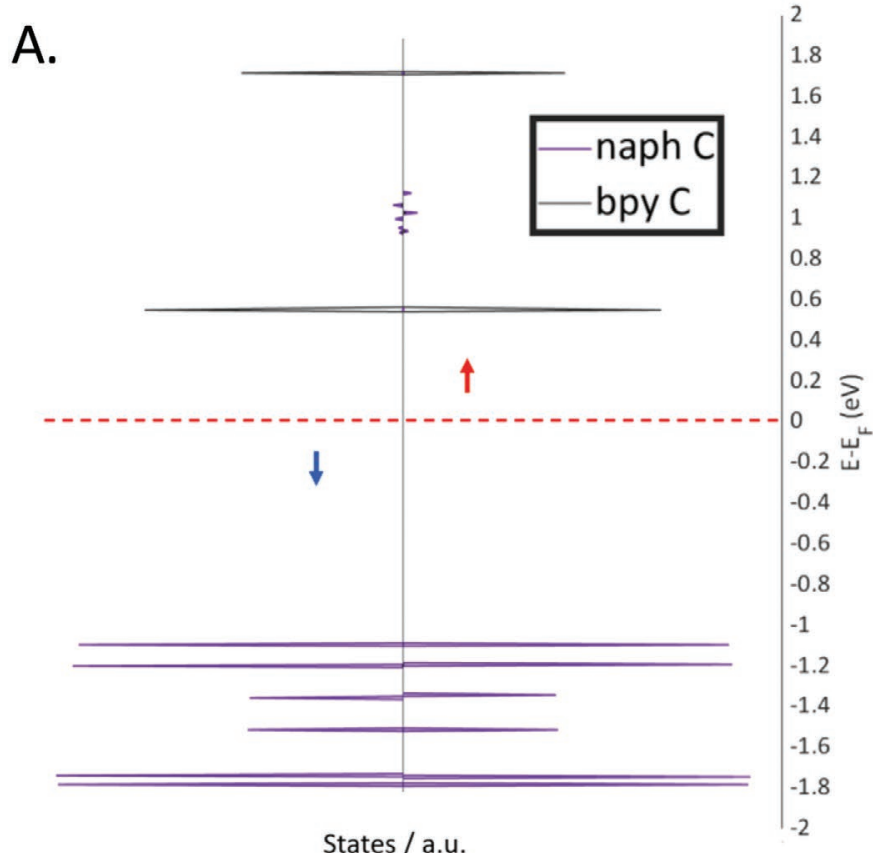

B.

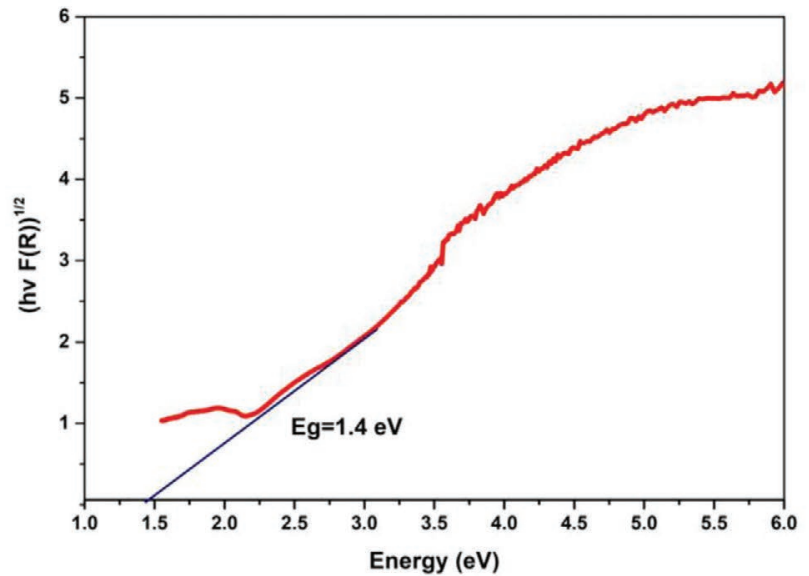

Figure 3. A) Spin-up and spin-down projected density of states of the 1,4-NDPA and 4,4'-bpy carbons for TUB75 in the AFM configuration. B) Indirect Tauc plot derived from the UV-Vis absorption spectrum of TUB75 (shown in Figure S2, Supporting Information), revealing a typical semiconductor pattern and a bandgap of $E_{\mathrm{g}}=1.4 \mathrm{eV}$.

magnetization) that increases with increasing field strength, a behavior that is characteristic of a material with AFM correlations (see Figure 5 for a depiction of the AFM correlations of spins in the geometry-optimized structure). This is corroborated by our DFT-calculated exchange energy (i.e., the energy difference between the AFM and FM configurations) of $E_{\mathrm{ex}}=E_{\mathrm{AFM}}-E_{\mathrm{FM}}=-37.3 \mathrm{meV}$, indicating that the AFM configuration is more stable than the FM one. Moreover, the position of the maximum does not vary with temperature, which is characteristic of the presence of short-range order in the 1D spin chains and is consistent with the zigzag chains observed in the crystal structure (Figure 1B). At $2 \mathrm{~K}$, we observe a non-zero magnetization that increases with increasing field strength and appears to plateau at higher fields; for the lower field strengths, the magnetization initially decreases and then increases with increasing temperature (up to $10 \mathrm{~K}$ ), while for the higher field strengths the magnetization simply increases with increasing temperature (up to $10 \mathrm{~K}$ ). The upturn of the magnetization below $10 \mathrm{~K}$ (known as a Curie tail) observed for the lower field strengths is suggestive of the presence of a small amount of paramagnetic impurity, e.g., $\mathrm{Cu}$ ions that are not embedded in the TUB75 crystal structure. ${ }^{[31]}$ At high temperatures, the magnetization decreases to zero with increasing temperature, as expected (see Figure 4A).

Given the underlying 1D chain geometry, we fit our hightemperature $(>30 \mathrm{~K})$ magnetic susceptibility data (Figure 4B) to Heisenberg chain and dimer chain models (depicted in Figure 4D). As shown in Figure 4B, close fits to the data were obtained with coupling constants of $J_{\text {chain }}=16.8 \mathrm{~K}$ and $J^{\prime}=-22 \mathrm{~K}$ for the Heisenberg chain model, ${ }^{[42,43]}$ and coupling constants of $J_{\text {dimer }}=54 \mathrm{~K}$ and $J_{\text {chain }}=-2.6 \mathrm{~K}$ for the dimer chain model. ${ }^{[4-46]}$ The signs and magnitudes of the Heisenberg chain coupling constants are consistent with those observed in antiferromagnetically coupled 1D chains, while those of the dimer chain model are suggestive of another type of coupling. The diamagnetic contribution is small in both cases. As the temperature is lowered to 1 from $30 \mathrm{~K}$, the magnetic susceptibility predicted by the dimer chain model drops rapidly (ultimately to negative values), in contrast with the experimental susceptibility which drops less rapidly and remains positive. On the other hand, the magnetic susceptibility predicted by the Heisenberg chain model drops less rapidly than the experimental susceptibility and remains positive. As mentioned earlier, these deviations may be due to the presence of impurity spins; thus, we fit the low-temperature $(<10 \mathrm{~K})$ magnetic susceptibility data to a field-dependent Brillouin function plus a baseline signal (dotted black line) that is due to the 1D chains. From Figure $4 \mathrm{~B}$, we see that this combination of functions (with an impurity content of $\approx 8 \%, S=12$ and $g=2$ ) closely fits the low-temperature data. Given that the magnetic susceptibilities of TUB75, the Heisenberg chain, and the dimer chain are positive, positive, and negative, respectively, at very low temperatures (see Figure 4B), it appears that its IBU is best described in terms of Heisenberg chains. A Curie-Weiss fit to the high temperature portion of the inverse magnetic susceptibility is shown in Figure 4C. All details of the data fitting are given in the Supporting Information.

Finally, in Figure 6, we present the DFT-calculated spin density isosurface, focusing on a portion of the IBU in the AFM configuration. The antiparallel spin density along the copper dimer chain is clearly seen and it is delocalized onto the coppers and their nearest-neighbor oxygens and 


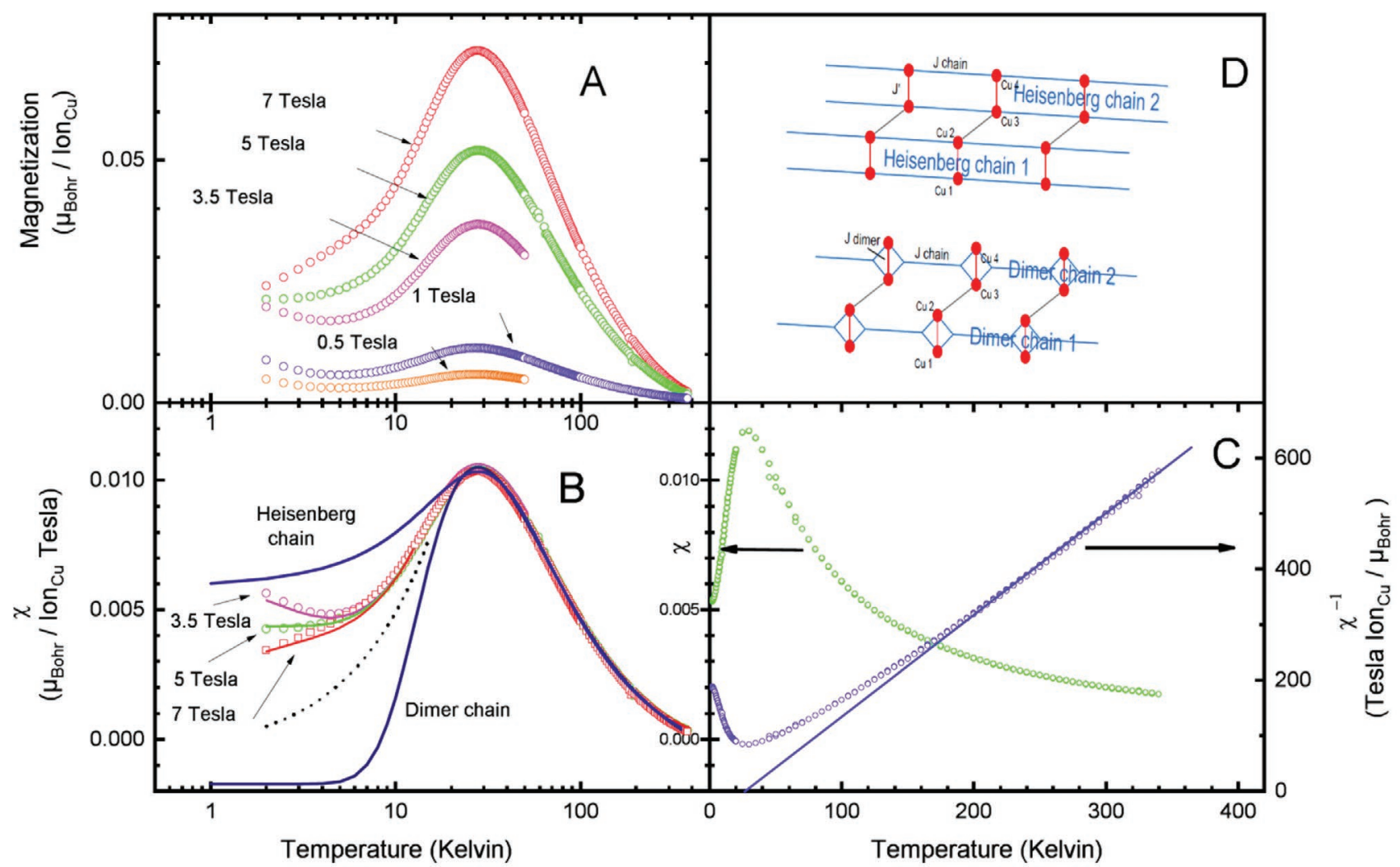

Figure 4. Magnetic response data for TUB75. A) Magnetization versus temperature data for TUB75 in different applied magnetic fields. B) Magnetic susceptibility, $\chi$, (colored circles) obtained from the magnetization data along with fits (solid blue lines) to the Heisenberg chain and dimer chain models. The upturn in the low-temperature signal $(<10 \mathrm{~K})$, which is suggestive of the presence of paramagnetic impurities, is fit by Brillouin functions (solid colored lines) with a baseline signal (dotted black line). C) Magnetic susceptibility (green circles) obtained in a 5 T magnetic field, corrected for the diamagnetic background, and the inverse susceptibility (purple circles) on which a Curie-Weiss high-temperature linear fit is shown (solid purple line). D) Schematic pictures of Heisenberg chains and dimer chains, the models used to fit the magnetic susceptibility data. See Supporting Information for details.

nitrogens. This result suggests that the magnetic behavior of TUB75 is likely dependent on the shared spin density of these three atoms.

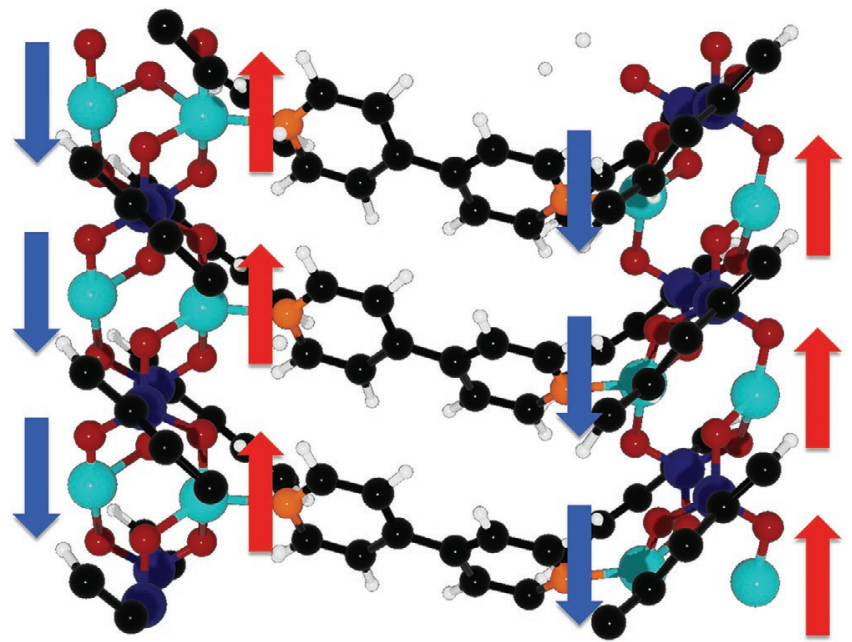

Figure 5. Minimum energy structure of the $1 \times 3 \times 1$ supercell depicting the antiferromagnetic configuration of the electrons on each copper $(\alpha-$ spin: red, $\beta$-spin: blue). Color definitions: $\mathrm{O}$-red; $\mathrm{N}$-orange; $\mathrm{Cu}-$ cyan; C-black; P-blue; $\mathrm{H}$-white.
Herein, we have reported on the conductive and magnetic properties of the phosphonate MOF, TUB75. With an experimental bandgap of $1.4 \mathrm{eV}$ and room-temperature (orientation-dependent) conductances ranging from $\approx 10^{-3}$ to $\approx 10^{3} \mathrm{~S} \mathrm{~m}^{-1}$, TUB75 is the first semiconducting phosphonate MOF in the literature, paving the way for a new family of semiconductors with an extremely rich structural chemistry. The metal-binding modes of the phosphonic acid group in TUB75 support a 1D IBU composed of a zigzag copper dimer chain, which was found to be antiferromagnetically coupled. The temperature-dependent magnetic susceptibility data was well fit using a combination of a Heisenberg chain model at higher temperatures and Brillouin functions at very low temperatures. Our experimental measurements were accompanied by DFT calculations, which yielded a bandgap of $1.77 \mathrm{eV}$ in good agreement with the experimental one and support the AFM nature of the IBU. Given the high thermal/chemical stabilities of phosphonate MOFs and the numerous metal-binding modes of phosphonates, our findings suggest that they could be used in next-generation electrodes and supercapacitors capable of withstanding harsh operating conditions. The vast structural diversity of phosphonate MOFs could lead to a new generation of porous materials with engineerable surface areas and magnetic/ conductive properties. Currently, we are working on the 


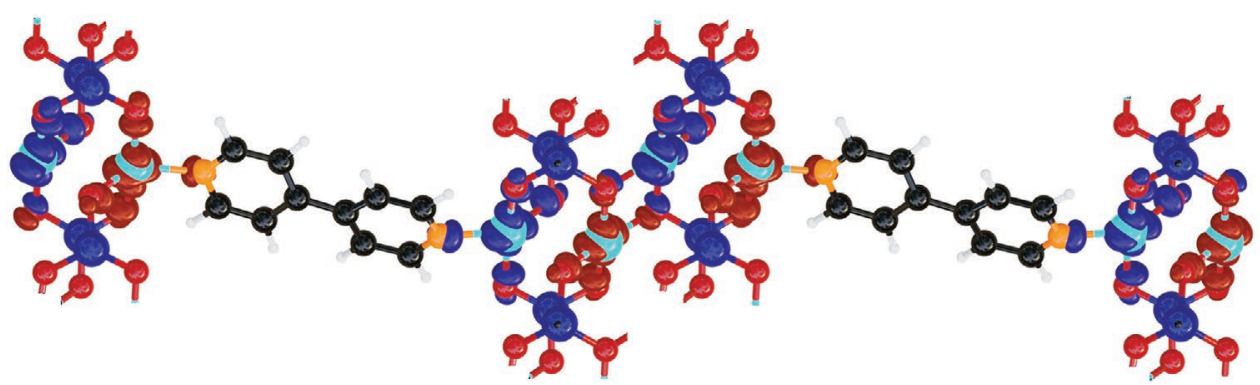

Figure 6. Spin density isosurface of a portion of the IBU. $\beta / \alpha$ spin density is shown in blue/red and corresponds to a difference between the spin-up and spin-down density of 0.005 electrons per $\AA^{3}$. Color definitions: O—red; $\mathrm{N}$-orange; $\mathrm{Cu}$-cyan; $\mathrm{C}$-black; $\mathrm{P}$-blue; $\mathrm{H}$-white.

reticular chemistry of phosphonate MOFs to explore these possibilities.

\section{Supporting Information}

Supporting Information is available from the Wiley Online Library or from the author.

\section{Acknowledgements}

The authors thank Dr. Pradip Pachfule from TU-Berlin for his help with the UV-vis measurements. G.Y. thanks the DFG for funding his work with grant number DFG YU 267/2-1 and DAAD for supporting Prof. Dr. Bünyemin Çoşut's visit to his lab at TU-Berlin. G.H. acknowledges support from the Natural Sciences and Engineering Research Council of Canada (NSERC). The DFT calculations were enabled by support provided by WestCrid (www. westgrid.ca) and Compute Canada (www.computecanada.ca).

\section{Conflict of Interest}

G.Y. has a patent pending protecting some of the presented results.

\section{Author Contributions}

K.S. and C.P. contributed equally to this work. G.Y. created the hypothesis, supervised the project, and wrote, synthesized, and revised the non-computational parts of the manuscript. P.T. generated the crystal structure figure (Figure 1) and resynthesized the crystals for the electrical conductivity measurements. C.A.P. performed the DFT calculations, generated the computational figures/tables, and wrote the initial drafts of the computational parts of the manuscript. G.H. supervised the calculations, wrote/revised the computational parts of the manuscript, and performed extensive critical revisions of the entire manuscript. K.S. performed the magnetic measurements and wrote the initial drafts of the corresponding methods and results sections. B.Ç. prepared the Tauc plot (Figure 3B). F.-J.S. performed the electrical conductivity measurements.

\section{Keywords}

DFT calculations, electrodes, magnetic MOFs, metal-organic frameworks, semiconductive MOFs, supercapacitors

Received: January 20, 2020

Revised: March 21, 2020

Published online: May 6, 2020
[1] M. Eddaoudi, J. Kim, N. Rosi, D. Vodak, J. Wachter, M. O'Keeffe, O. M. Yaghi, Science 2002, 295, 469.

[2] H. C. Zhou, J. R. Long, O. M. Yaghi, Chem. Rev. 2012, 112, 673.

[3] O. M. Yaghi, M. O'Keeffe, N. W. Ockwig, H. K. Chae, M. Eddaoudi, J. Kim, Nature 2003, 423, 705.

[4] H. Furukawa, K. E. Cordova, M. O'Keeffe, O. M. Yaghi, Science 2013, $341,1230444$.

[5] H.-C. "Joe" Zhou, S. Kitagawa, Chem. Soc. Rev. 2014, 43, 5415.

[6] M. J. Kalmutzki, N. Hanikel, O. M. Yaghi, Sci. Adv. 2018, 4, eaat9180.

[7] A. Schoedel, M. Li, D. Li, M. O'Keeffe, O. M. Yaghi, Chem. Rev. 2016, 116, 12466.

[8] S. M. Cohen, J. Am. Chem. Soc. 2017, 139, 2855.

[9] Z. Wang, S. M. Cohen, J. Am. Chem. Soc. 2007, 129, 12368.

[10] K. K. Tanabe, S. M. Cohen, Chem. Soc. Rev. 2011, 40, 498.

[11] M. T. Kapelewski, T. Runčevski, J. D. Tarver, H. Z. H. Jiang, K. E. Hurst, P. A. Parilla, A. Ayala, T. Gennett, S. A. Fitzgerald, C. M. Brown, J. R. Long, Chem. Mater. 2018, 30, 8179.

[12] M. Ding, R. W. Flaig, H. L. Jiang, O. M. Yaghi, Chem. Soc. Rev. 2019, 48, 2783.

[13] P. Horcajada, R. Gref, T. Baati, P. K. Allan, G. Maurin, P. Couvreur, G. Férey, R. E. Morris, C. Serre, Chem. Rev. 2012, 112, 1232.

[14] K. J. Hartlieb, D. P. Ferris, J. M. Holcroft, I. Kandela, C. L. Stern, M. S. Nassar, Y. Y. Botros, J. F. Stoddart, Mol. Pharmaceutics 2017, 14, 1831.

[15] S. Rojas, T. Baati, L. Njim, L. Manchego, F. Neffati, N. Abdeljelil, S. Saguem, C. Serre, M. F. Najjar, A. Zakhama, P. Horcajada, J. Am. Chem. Soc. 2018, 140, 9581.

[16] M. Ranocchiari, J. A. Van Bokhoven, Phys. Chem. Chem. Phys. 2011, 13, 6388.

[17] A. Dhakshinamoorthy, Z. Li, H. Garcia, Chem. Soc. Rev. 2018, 47, 8134.

[18] E. Coronado, G. Mínguez Espallargas, Chem. Soc. Rev. 2013, 42, 1525.

[19] G. Mínguez Espallargas, E. Coronado, Chem. Soc. Rev. 2018, 47, 533.

[20] E. Coronado, M. Giménez-Marqués, G. M. Espallargas, L. Brammer, Nat. Commun. 2012, 3, 828 .

[21] C. Yang, R. Dong, M. Wang, P. S. Petkov, Z. Zhang, M. Wang, P. Han, M. Ballabio, S. A. Bräuninger, Z. Liao, J. Zhang, F. Schwotzer, E. Zschech, H. H. Klauss, E. Cánovas, S. Kaskel, M. Bonn, S. Zhou, T. Heine, X. Feng, Nat. Commun. 2019, 10, 3260.

[22] L. Sun, M. G. Campbell, M. Dincă, Angew. Chem., Int. Ed. 2016, 55, 3566.

[23] D. Sheberla, J. C. Bachman, J. S. Elias, C. J. Sun, Y. Shao-Horn, M. Dincǎ, Nat. Mater. 2017, 16, 220.

[24] S. I. Ohkoshi, H. Tokoro, Acc. Chem. Res. 2012, 45, 1749.

[25] W. Ouellette, A. V. Prosvirin, K. Whitenack, K. R. Dunbar, J. Zubieta, Angew. Chem., Int. Ed. 2009, 48, 2140.

[26] T. B. Faust, D. M. D'Alessandro, RSC Adv. 2017, 4, 17498. 
[27] D. Maspoch, D. Ruiz-Molina, K. Wurst, N. Domingo, M. Cavallini, F. Biscarini, J. Tejada, C. Rovira, J. Veciana, Nat. Mater. 2003, 2, 190.

[28] O. K. Farha, I. Eryazici, N. C. Jeong, B. G. Hauser, C. E. Wilmer, A. A. Sarjeant, R. Q. Snurr, S. T. Nguyen, A. Ö. Yazaydin, J. T. Hupp, J. Am. Chem. Soc. 2012, 134, 15016.

[29] G. Yücesan, V. Golub, C. J. O'Connor, J. Zubieta, Solid State Sci. 2005, 7, 133.

[30] M. A. AlDamen, S. Cardona-Serra, J. M. Clemente-Juan, E. Coronado, A. Gaita-Ariño, C. Martí-Gastaldo, F. Luis, O. Montero, Inorg. Chem. 2009, 48, 3467.

[31] D. T. Tran, X. Fan, D. P. Brennan, P. Y. Zavalij, S. R. J. Oliver, Inorg. Chem. 2005, 44, 6192.

[32] K. J. Gagnon, H. P. Perry, A. Clearfield, Chem. Rev. 2012, 112, 1034.

[33] M. Taddei, F. Costantino, R. Vivani, Eur. J. Inorg. Chem. 2016, 27, 4300.

[34] G. Yücesan, Y. Zorlu, M. Stricker, J. Beckmann, Coord. Chem. Rev. 2018, 369, 105.

[35] Y. Zorlu, D. Erbahar, A. Çetinkaya, A. Bulut, T. S. Erkal, A. O. Yazaydin, J. Beckmann, G. Yücesan, Chem. Commun. 2019, 55, 3053.
[36] S. J. I. Shearan, N. Stock, F. Emmerling, J. Demel, P. A. Wright, K. D. Demadis, M. Vassaki, F. Costantino, R. Vivani, S. Sallard, I. R. Salcedo, A. Cabeza, M. Taddei, Crystals 2019, 9, 270.

[37] R. A. Coxall, S. G. Harris, D. K. Henderson, S. Parsons, P. A. Tasker, R. E. P. Winpenny, J. Chem. Soc., Dalton Trans. 2000, 14, 2349.

[38] A. Bulut, Y. Zorlu, M. Wörle, A. Çetinkaya, H. Kurt, B. Tam, A. Ö. Yazaydın, J. Beckmann, G. Yücesan, ChemistrySelect 2017, 2, 7050.

[39] A. Bulut, M. Maares, K. Atak, Y. Zorlu, B. Çoşut, J. Zubieta, J. Beckmann, H. Haase, G. Yücesan, CrystEngComm 2018, 20, 2152.

[40] A. R. Zanatta, I. Chambouleyron, Phys. Rev. B 1996, 53, 3833.

[41] J. Tauc, R. Grigorovici, A. Vancu, Phys. Status Solidi B 1966, 15, 627.

[42] W. E. Hatfield, R. R. Weller, J. W. Hall, Inorg. Chem. 1980, 19, 3825.

[43] J. C. Bonner, M. E. Fisher, Phys. Rev. 1964, 135, A640.

[44] D. C. Johnston, in Handbook of Magnetic Materials, Vol. 10 (Ed: K. H. J. Buschow), Elsevier, Amsterdam, The Netherlands 1997.

[45] E. S. Klyushina, A. T. M. N. Islam, J. T. Park, E. A. Goremychkin, E. Wheeler, B. Klemke, B. Lake, Phys. Rev. B 2018, 98, 104413.

[46] S. Eggert, I. Affleck, M. Takahashi, Phys. Rev. Lett. 1994, 73, 332. 